\title{
Typologie exploratoire des affordances textuelles
}

Éric Kavanagh, Jacynthe Roberge et Isabelle Sperano

\section{(2) OpenEdition}

\section{Journals}

Édition électronique

URL : http://journals.openedition.org/pratiques/3187

DOI : 10.4000/pratiques.3187

ISSN : 2425-2042

Éditeur

Centre de recherche sur les médiations (CREM)

\section{Référence électronique}

Éric Kavanagh, Jacynthe Roberge et Isabelle Sperano, « Typologie exploratoire des affordances textuelles », Pratiques [En ligne], 171-172 | 2016, mis en ligne le 07 février 2017, consulté le 10 décembre 2020. URL : http://journals.openedition.org/pratiques/3187 ; DOI : https://doi.org/10.4000/ pratiques. 3187

Ce document a été généré automatiquement le 10 décembre 2020.

(C) Tous droits réservés 


\title{
Typologie exploratoire des affordances textuelles
}

\author{
Éric Kavanagh, Jacynthe Roberge et Isabelle Sperano
}

\section{Introduction}

1 L'avancement des connaissances en rédactologie passe notamment par une meilleure compréhension de l'activité de lecture et, conséquemment, par un enrichissement des modèles existants. Au-delà de la dimension psycholinguistique, déjà assez bien comprise (Marin et Legros, 2008; Caron, 2008), la lecture est un phénomène complexe qui s'enclenche bien avant le traitement psycholinguistique à proprement parler et qui suppose également un traitement en parallèle de ce dernier. Bien sûr, on pourra débattre de la justesse d'employer le terme lecture pour décrire la gamme de ces activités pré- et parapsycholinguistiques, mais là n'est pas exactement notre propos. Si les activités perceptuelles que nous nous apprêtons à décrire n'entrent pas dans la définition stricte de la lecture, elles font néanmoins partie de son continuum et, en ce sens, en sont indissociables, surtout si la rédactologie aspire à mieux comprendre l'interaction du lecteur avec son texte. Si nous voulons jeter un regard sur l'activité de lecture prise dans un cadre un peu plus large que celui de la psycholinguistique (cadre tout à fait légitime que nous ne remettons en cause d'aucune façon ici), c'est que nous désirons ouvrir de nouvelles perspectives sur le texte.

2 L'approche que nous proposons invite à considérer le texte non pas seulement ni d'abord comme un matériau linguistique mais bien comme un objet du monde avec lequel interagit un humain-utilisateur-lecteur, et cela, à tous les instants de l'interaction. Présenté sous forme de typologie exploratoire - ce qui trahit son stade d'avancement encore bien modeste -, notre modèle en devenir suppose, à certains niveaux plus profonds, le passage rapide et fréquent entre un traitement psycholinguistique du texte et un autre type de traitement dont il sera question ici. Nous croyons qu'un tel enrichissement du modèle par la prise en compte d'un traitement parallèle au traitement 
psycholinguistique (et plus global que ce dernier) permettrait, d'une part, pour les théoriciens, de mettre à jour un mécanisme central de guidage pour la lecture (une forme de programmation de la lecture) et, d'autre part, pour les praticiens, de mieux cibler l'intervention rédactionnelle/conceptuelle en l'appuyant sur un modèle qui décrit avec plus de complétude l'interaction lecteur-texte ou l'interaction utilisateur-document. Les traitements à survenir avant le traitement psycholinguistique et en parallèle de ce dernier sont sans doute nombreux - et ne s'accommodent certainement pas tous du terme traitement (pensons, par exemple, à l'impact des émotions, de la motivation, etc.). Dans le cadre de notre modèle en devenir, il nous est apparu prioritaire de considérer un type de traitement en lien direct avec le passage à l'action : la perception des affordances.

3 L'examen des différentes affordances textuelles (et du document) pourrait mettre en évidence des caractéristiques de l'objet à lire qu'on a sous-estimées parce qu'elles passent tout simplement sous le radar des disciplines et des cadres théoriques habituellement convoqués pour étudier les produits de la rédaction professionnelle, leur utilisation et leur compréhension. Sans du tout évoquer les affordances, Dillon a déjà fait état de la limite de certains modèles dominants pour décrire le processus de lecture.

There are few psychological models of reading that consider test manipulation or navigation, for example, as part of the reading process. [...] Can any view that claims reading only starts when the "eye meets the page" really lay claim to cover the "entire" process? Certainly it appears logical to start here, but if the situation demands that the reader moves from text to text in their search for information, do we conclude that the intervening moves are not part of the process of reading? (Dillon, $2004: 71$ )

Dans la suite du texte, nous présentons d'abord le concept d'affordance et un survol de son évolution, de son cadre psychologique d'origine jusqu'à son transfert et à son utilisation actuelle en design de communication, domaine très proche de la rédaction professionnelle à plusieurs égards (conception et production d'artéfacts communicationnels, universalité de la triade concepteur-client-utilisateur, etc.). Ensuite, nous exposerons nos arguments en appuyant le transfert du concept d'affordance vers la rédactologie. Enfin, nous présenterons une typologie exploratoire des affordances pertinentes pour la rédactologie.

\section{Les affordances}

Les affordances (de l'anglais affordance, du verbe to afford "permettre ») peuvent être définies comme des propriétés de l'environnement et des objets qui s'y trouvent, propriétés qui, lorsqu'elles sont perçues, permettent à l'animal - à l'humain - de savoir comment il peut agir (se déplacer, se cacher, s'agripper, saisir quelque chose, etc.) dans un contexte donné. «Les affordances sont déterminées conjointement par les caractères physiques d'un objet et par les capacités sensorielles, motrices et mentales d'un être vivant » (OQLF-GDT, 1995). Les affordances sont traitées de façon automatique (automatic perceptual process), « sous la barre » de la conscience, c'est-à-dire sans qu'il y ait décodage au sens de l'approche de traitement de l'information (Fortin \& Rousseau, 2012). Cette caractéristique est centrale dans la mesure où les affordances sont perçues indépendamment de la volonté et constitue donc un niveau de traitement parallèle au traitement de l'information et qui peut interférer avec lui de manière diverse. La nature des affordances varie selon le point de vue et le profil de l'observateur : par exemple, en contemplant le fauteuil devant lui, un chaton perçoit un objet du monde qu'il peut 
escalader ou sur lequel il peut sauter alors que l'humain adulte percevra un élément de l'environnement sur lequel il peut s'asseoir. De même, regardant un bloc de ciment déposé sur le sol, l'humain adulte pourra percevoir un objet qu'il peut ou non saisir, soulever et apporter avec lui alors que le rongeur de petite taille y percevra les affordances d'une cachette idéale pour se protéger de ses prédateurs. Pour un même objet du monde, la potentialité d'action(s) diffère selon l'animal concerné et le point de vue.

Comme le traitement des affordances est automatique, ininterrompu et omniprésent, nous postulons qu'il s'applique aussi aux objets du monde destinés à être lus. Et, en ce sens, les affordances doivent intéresser la rédactologie parce qu'elles influencent à divers degrés les interactions avec les documents et les textes en circulation dans notre société, quel que soit le contexte.

\section{3. Évolution du concept d'affordance}

7 Le concept d'affordance a été proposé par James J. Gibson dans le cadre de sa psychologie écologique $(1979)^{1}$. Il décrit alors les affordances de la façon suivante :

The affordances of the environment are what it offers the animal, what it provides or furnishes, either for good or ill. The verb to afford is found in the dictionary, but the noun affordance is not. I have made it up. (Gibson, 1979 :119)

Plus loin, il poursuit en donnant l'un des exemples les plus cités et les plus fondamentaux de sa théorie :

If a terrestrial surface is nearly horizontal (instead of slanted), nearly flat (instead of convex or concave), and sufficiently extended (relative to the size of the animal) and if its surface is rigid (relative to the weight of the animal), than the surface affords support. (Gibson, 1979 : 119)

9 Il en va de même pour tous les éléments de l'environnement, qu'ils soient fixes ou mobiles.

10 Décédé en 1979, quelques mois après la publication de son ouvrage le plus connu (1979), Gibson n'a pas vraiment développé sa théorie des affordances au-delà de l'exposé introductif qu'il propose dans ce dernier. Toutefois, une cohorte de chercheurs - parmi lesquels on compte Eleanor Gibson (2000), son épouse - ont poursuivi la réflexion, notamment dans la revue scientifique Ecological Psychology (depuis 1989). Le statut de l'affordance a, depuis, fait et fait encore couler beaucoup d'encre dans la communauté de chercheurs en psychologie écologique (Chemero, 2003; Stoffregen, 2003, 2004; Michaels, 2003; Jones, 2003).

11 C'est en 1988, dans son ouvrage phare, que Donald A. Norman introduit le concept d'affordance à la communauté des designers et des praticiens en interaction humainmachine (IHM).

Affordances provide strong clues to the operation of things. Plates are for pushing. Knobs are for turning. Slots are for inserting things into. Balls are for throwing or bouncing. When affordances are taken advantages of, the user knows what to do just by looking: no picture, label, or instruction is required. (Norman, $2002: 9)$

12 Enseigné et présenté dans plusieurs spécialités du design telles que le design de produits et d'ingénierie (Maier, 2011; Gero et Kannengiesser, 2012) et le design de communication (Norman, 2002; Kaptelinin et Nardi, 2012a), le concept d'affordance gagnera rapidement en popularité et sera intégré à de grands lexiques du domaine ainsi qu’à plusieurs 
inventaires de principes de design (APCI et al., 2013; Erlhoff et Marshall, 2008; Lidwell, Holden \& Butler, 2003, 2015; Soegaard, 2003; Weinschenk, 2011). Cette popularité semble avoir été engendrée notamment par cet assouplissement de Norman à l'égard de la définition gibsonienne de l'affordance: "I believe that affordances result from the mental interpretation of things, based on our past knowledge and experience applied to our perception of the things about us. » (Norman, 2002: 219).

Mais cette ouverture vers l'affordance «apprise " n'est pas alignée sur l'esprit du paradigme gibsonien, et Norman (2002) le reconnait : « My view is somewhat in conflict with the views of many Gibsonian psychologists, but this internal debate within modern psychology is of little relevance here. » (Norman, 2002 : 119). Pourtant, dix ans plus tard, Norman reproche aux designers d'interface (parfois avec virulence) un emploi abusif du concept d'affordance et, presque ironiquement, reconnait sa responsabilité dans cet abus. Parlant de l'usage du concept en design, il dira :

Alas, yes, the concept has caught on, but not always with complete understanding. My fault: I was really talking about perceived affordances, which are not at all the same as real ones. [...] I will make a global change [dans une prochaine édition de l'ouvrage], replacing all instances of the word "affordance" with the phrase "perceived affordance." The designer cares more about what actions the user perceives to be possible than what is true. Moreover, affordances, both real and perceived, play very different roles in physical products than they do in the world of screen-based products. In the latter case, affordances play a relatively minor role: cultural conventions are much more important. (Norman, 1999 : 39)

Pour Norman, ce que plusieurs designers traitent comme des affordances (ex. : le bouton cliquable à l'écran) relève en fait des conventions culturelles (apprises) et non de la perception directe, principe central de la psychologie gibsonienne, caractéristique des affordances.

\section{Comment convoquer la théorie des affordances en rédactologie?}

15 Cette critique de Norman à l'endroit des affordances perçues à l'écran - et, par extension, des affordances perçues sur papier ou sur tout support scriptural - venait pratiquement interdire d'associer le concept d'affordance aux aspects langagiers. Mais deux contributions scientifiques allaient fournir l'ancrage et le tremplin nécessaire pour engager notre réflexion à ce propos.

D'une part, lors d'une relecture de Stoffregen (2004), nous avons été intrigués par un exemple qu'il présente et qui ne nous avait pas marqués auparavant:

I believe that under my definition, affordance is a tightly limited concept. If affordance includes all the things that an animal (or set of animals) can do in a given situation or environment, then it excludes the uncountably large number of things that the animal (or set of animals) cannot do in that situation or environment. Because I am fluent in English, I can read books in English, but because I am ignorant of Russian, I cannot read books in that language. [...] Thus, my definition of affordance provides considerable heuristic guidance in the search for animal and environmental properties whose relation gives rise to opportunities for action. (Stoffregen, $2004: 84-85$ )

17 Cet exemple de Stoffregen, - qu'on peut reformuler de la façon suivante : détecter une langue que je connais permet (afford) la lecture ou la conversation dans cette langue - nous 
autorisait à poursuivre la réflexion sur les affordances du matériel linguistique (au sens large).

D'autre part, le modèle de Still et Dark (2013) - récent par ailleurs - allait nous permettre de recadrer le concept de convention culturelle imposé comme limite forte par Norman, dans la mesure où les auteurs introduisent un autre critère de définition de l'affordance :

Traditionally, affordances were discussed within a Gibsonian framework in which affordances arise from direct perception. Some authors now describe affordances as being mostly perceptual while others describe them as being culturally bound. We suggest that both of these descriptions are correct and that they can be explained from a cognitive conceptualization of perceived affordances. We suggest that perceived affordances are supported by automatic perceptual processes in the user developed over time through consistent interactions with the environment. [...]. We propose that when a design produces highly consistent interactions, the cognitive pattern recognition system learns to automatically identify the constraints. We are not interested in any specific type of constraint (e.g., physical, social, logical, cultural, semantic); rather, we are interested in the cognitive effect of a constraint's being in play. (Still \& Dark, 2013 : 285)

Sans entrer dans le détail de la mécanique des contraintes et de l'automatisation, nous retenons le modèle de Still et Dark parce qu'il désigne clairement comme affordances perçues des éléments qui ont d'abord été appris (ex. : conventions d'un genre) et dont la reconnaissance est désormais passée en perception directe et, donc, automatique. En guise de caution intellectuelle et scientifique, Norman lui-même aurait commenté positivement le modèle (Still \& Dark, 2013: 299), ce qui constitue un appui particulièrement pertinent compte tenu de l'histoire du concept. Suivant cela, nous estimons être autorisés à considérer les objets rédactionnels dans une réflexion sur l'affordance.

\section{Typologie des affordances pertinentes en rédactologie}

20 La nature des affordances fait en sorte qu'on ne peut s'y abstraire lorsqu'elles sont perçues (Still \& Dark, 2013). Elles déclenchent spontanément la sélection de certains schémas mentaux ou entraînent des (micro) comportements automatisés. Omniprésentes, dans le monde et les objets du monde, les affordances sont donc aussi contenues dans tous les artéfacts destinés à la lecture (au sens large), qu'il s'agisse d'un roman, d'un panneau publicitaire, d'une page Web, du menu dans une application mobile, d'un formulaire fiscal, etc.

Compte tenu de la nature des affordances et du fait qu'elles diffèrent en fonction de l'être qui perçoit et du point de vue adopté, il semble impossible d'établir une liste exhaustive des affordances, même pour un contexte restreint et très bien décrit. Cependant, dans une logique exploratoire, nous avons identifié six types d'affordances qui nous semblent caractériser tous les documents/textes susceptibles d'exister dans le monde de l'imprimé et du numérique. Les types proposés s'inscrivent dans une logique progressive descendante, c'est-à-dire dans un mouvement perceptuel allant des affordances macroscopiques (qu'on perçoit à distance de l'objet communicationnel) vers les affordances microscopiques (qui nécessitent d'« entrer» dans le document et dans le texte), mais toujours en marge du processus de lecture psycholinguistique à proprement parler. Malgré ce mouvement (du macro vers le micro), il est possible que 
certaines affordances de types différents soient perçues en concomitance ou dans un ordre inverse.

\subsection{Affordances gibsoniennes (ou de l'objet)}

Ce premier niveau renvoie au sens premier de l'affordance (Gibson, 1979). Tout texte produit est incarné dans un objet du monde réel, un objet qui peut prendre une infinité de formes et d'aspects (ex. : livre, écran, panneau, etc.). Il n'y a pas de lecture possible sans interaction avec ces objets du monde. Même si l'interaction est inconsciente, très subtile ou encore très rapide, l'objet porteur ou vecteur du texte constitue néanmoins une certaine frontière à franchir avant d'atteindre le texte. En ce sens, l'interaction avec l'objet porteur du texte fait partie du processus de lecture au sens large, et cela, à des degrés divers selon les caractéristiques de l'objet et la nature de l'activité de lecture. Pensons au livre, que l'on saisit, feuillette, transporte, dépose, ou encore aux textes diffusés sur le Web que l'on manipule avec une souris, un clavier, un écran. Notons que la potentialité d'action perçue ne correspond pas toujours à un usage culturellement normé. En ce sens, un enfant ne sachant pas lire peut très bien percevoir ces affordances de premier niveau et utiliser l'objet à d'autres fins que la lecture (ex. : mordiller le coin d'un livre pour soulager un mal de dents, dessiner un soleil sur un formulaire de déclaration de revenus, etc.). Si certains textes ne nécessitent aucune manipulation physique comme telle (ex.: message sur un panneau publicitaire, message inscrit dans le sable, graffiti, signalisation routière), ils sont néanmoins des objets de l'environnement susceptibles de modifier le comportement des humains qui entrent en contact, même distant, avec eux.

Traditionnellement, la conception et la production de ces objets du monde ont rarement été la responsabilité première du rédacteur professionnel. C'est le plus souvent à un designer graphique, d'interface, de produits ou même à un ingénieur que revient la tâche de concevoir ces objets. Toutefois, étant donné que les décisions prises au moment de la conception du produit (ex. : choix de matériaux, gestion des contraintes ergonomiques, sélection et mise en forme du contenu, etc.) auront des impacts parfois majeurs sur l'utilisabilité de l'objet et, par conséquent, des impacts sur la lecture elle-même, le rédacteur doit se préoccuper de cet aspect de la conception de l'objet porteur du texte.

À ce niveau de notre typologie, le rédacteur doit s'assurer que l'objet du monde vecteur de son texte soit d'abord et avant tout accessible à la perception et qu'il projette les affordances des actions voulues et cohérentes avec l'objectif de communication visé. Les caractéristiques de l'objet doivent permettre (to afford) le mode de lecture anticipé et planifié. Ainsi, par exemple, on attendra la pliabilité optimale d'un dépliant à 12 volets ou de la carte géotouristique, l'utilisabilité sans faille d'un site Web, la visibilité de la signalisation dans un hôpital, l'accessibilité des instructions d'un concours sur les gobelets à café d'une grande chaîne, etc. Évidemment, dans le cas où l'objectif de communication serait de confondre le lecteur (ex.: dans un contexte artistique ou humoristique), on pourrait, à l'inverse, veiller à brouiller certaines affordances de l'objet de manière à atteindre l'effet désiré.

\subsection{Affordances de lisibilité fondamentale}

Les affordances gibsoniennes concernent l'objet physique porteur du texte mais ne concernent pas le texte en lui-même. Ce n'est qu'à un deuxième niveau perceptuel que 
des affordances d'une autre nature viennent révéler le caractère lisible d'une partie de cet objet du monde. À ce niveau, l'objet est porteur d'indices susceptibles d'informer l'humain d'une potentialité d'action/interaction très précise qu'on nomme lecture. Ce deuxième niveau d'affordances ne concerne ni le décodage des formes linguistiques en présence ni non plus l'accès au sens lexical ni encore moins la compréhension partielle ou globale du texte. Ces affordances perceptibles ne renvoient que le message suivant: cet objet est destiné à la lecture.

Un enfant ne sachant pas lire mais conscient de l'activité de lecture pourra percevoir ces affordances et, on le voit très souvent, pourra même mimer l'acte de lecture en utilisant cet objet sans pourtant savoir décoder quelque forme linguistique que ce soit. Dans ce cas précis, l'enfant répond aux affordances de lisibilité fondamentale (ceci permet l'action de lire) sans savoir lire au sens strict. La polysémie du terme lisibilité (graphique, typographique, lexicale, textuelle, etc.) et la nécessité de l'employer par souci de clarté nous a amenés à nommer ce niveau affordances de lisibilité fondamentale. En effet, la première lisibilité possible implique une affordance à lire au sens le plus général et fondamental qui soit. Si le rédacteur (au sens strict) n'intervient habituellement pas à ce niveau d'affordance, le designer de documents doit toutefois se préoccuper de ce niveau perceptuel.

\subsection{Affordances linguistiques fondamentales}

27 Après avoir perçu les affordances d'un certain objet du monde et après avoir perçu qu'il s'agissait d'un objet lisible au sens le plus fondamental, l'humain doté d'une compétence minimale en lecture pourra percevoir que cet objet lisible au sens fondamental permet ou non la lecture dans une certaine modalité scriptovisuelle et, ultimement, dans un code ou une langue qu'il maîtrise en totalité ou en partie. Par exemple, un francophone confronté à un texte rédigé en français, en plus de reconnaître sa langue maternelle, reconnaîtra certaines caractéristiques du code alphabétique avec lequel est composé le texte, notamment ses graphèmes fondamentaux, sa bicaméralité (minuscules, majuscules), ses propriétés d'accentuation, sa direction (horizontale dextroverse dans le cas du français), les extensions des graphèmes (ex. : les digraphes $\propto$, ou, $c h$ ), etc. Même lorsqu'une langue n'est pas connue d'un lecteur, certaines caractéristiques du code scriptural peuvent permettre la lecture : par exemple, pour un francophone, ne pas comprendre le roumain ou le hongrois n'est pas un obstacle absolu à la lecture partielle d'un texte rédigé dans l'une ou l'autre de ces langues (ne pas confondre ici lecture du surface et compréhension) puisque le code alphabétique est partagé (sauf quelques exceptions). C'est d'autant plus vrai lorsqu'on compare ce que permet au francophone unilingue un texte composé en alphabet latin comparativement à un texte composé en alphabet géorgien, en syllabaire inuktitut, en linéaire B ou encore en sinogrammes.

\subsection{Affordances du genre}

D'un point de vue rédactologique, la question du genre d'un texte ou d'un document est déterminante à la fois en production et en réception. Le texte donné à lire n'est accessible qu'à travers les propriétés matérielles, graphiques et linguistiques en partie conventionnées (et à des degrés fort variables) par cet ensemble de représentations internalisées qu'on nomme genre et qui se présente dans des formes très variées, de la 
lettre à la page Web (Clerc \& Kavanagh, 2006). Consciemment ou non, les lecteurs/ utilisateurs programment en partie leur usage du document en tenant compte de ces conventions. Par exemple, les pages de résultats d'un moteur de recherche Web (Google autant que Facebook) présentent presque toujours une zone publicitaire que l'utilisateur régulier a appris à éviter du regard (Nielsen, 2007). Il le fait inconsciemment. Dans ces mêmes pages, l'internaute agit de façon automatique lorsqu'il porte son regard à la marge des trois premiers résultats sans égard à la pertinence réelle de ces résultats. Ces comportements (et bien d'autres) sont enclenchés à la suite de la perception de certaines affordances du genre. Moins un utilisateur est expérimenté, moins il perçoit les affordances du genre et plus il devra apprendre la structure et le fonctionnement du document. On peut déjà formuler l'hypothèse qu'un lecteur compétent l'est en partie parce qu'il est en mesure de percevoir les affordances pertinentes du genre (ce qui est vrai aussi pour tous les autres type d'affordances). Cet exemple nous permet aussi d'entrevoir l'existence d'affordances trompeuses ou fantômes, c'est-à-dire des affordances qui induisent le lecteur en erreur (ex. : la traitement scientifique de certaines publicités ou encore le traitement publicitaire de certains contenus plutôt neutres; le soulignement non hypertextuel dans le Web).

\subsection{Affordances des zones informationnelles}

Entre chaque épisode de lecture au sens strict, un lecteur/utilisateur adopte un comportement de balayage oculaire. Ce balayage est bien sûr guidé par des objectifs et des attentes (traitement descendant) mais il l'est aussi par des indices matériels (traitement ascendant) qui viennent influencer le parcours de lecture. Ainsi, à son entrée dans le document, le lecteur/utilisateur perçoit des indices qui révèlent l'existence de zones informationnelles plus ou moins formelles (ex. : titre, tableau, liste à puces, figure, photo, illustration, vidéo intégrée dans la page Web, système de renvoi ou de notes, paratextes, pagination, etc.). De façon automatique, le lecteur/utilisateur, expérimenté ou non, associera alors des fonctions particulières à ces différentes zones. Ce processus fonctionne en deux temps. D'abord, l'observateur perçoit les affordances de zonage, c'està-dire tous les indicateurs graphiques (ou autres) qui distinguent une zone du texte ou du document d'une autre zone. Ces affordances ne permettent pas seulement la reconnaissance du découpage mais bien l'association automatique suivante : un traitement distinct $=$ une zone distincte $=$ une fonction distincte présumée. Ensuite, à un deuxième niveau de perception automatique, l'observateur perçoit certaines affordances liées à la nature des zones préalablement perçues. Par exemple, la perception des affordances d'un paragraphe long peut déclencher une affordance de type ce paragraphe est très lourd et étendu et suppose une lecture longue et (peut-être) ardue et, découlant de cette perception, une démotivation immédiate pouvant causer l'abandon de la lecture.

En plus de ces affordances confinées à des éléments du découpage en zones informationnelles, le document peut contenir des affordances un peu plus diffuses, comme c'est le cas par exemple dans les textes techniques ou scientifiques, où la présence de chiffres, de pourcentages, de symboles mathématiques, etc., dans l'ensemble du document peut engendrer des comportements particuliers (ex.: éviter les passages perçus comme trop scientifiques pour accélérer la lecture). Dans une prochaine version du modèle, ces affordances diffuses pourraient constituer un type à part entière. 


\subsection{Affordances microstructurelles}

31 On retrouve les affordances microstructurelles à l'intraphrastique. Perçues en parallèle du traitement psycholinguistique et interférant avec lui, ces affordances peuvent être perçues dans la ponctuation (haute/basse/horizontale), la longueur de la phrase, l'emploi des noms propres ou de la majuscule, le soulignement ou tout autre traitement hypertextuel, la mise en évidence (gras, italique, couleur, soulignement, etc.), un traitement typographique particulier (appel, coupure de mots, exposant, etc.), un symbole rare ou inattendu, le retrait en début paragraphe, etc. Ces différents éléments peuvent contenir des affordances qui permettront des actions et des comportements de bifurcation (ex.: on clique sur l'hyperlien pour se rendre ailleurs), de rupture de la séquence normale de la lecture (ex. : saut du regard X lignes plus bas à cause de la présence de parenthèses, de tirets longs, d'un nom propre, etc.), de raccourcissement de la lecture (ex. : lecture du passage mis en évidence uniquement) et d'arrêt ou suspension de la lecture (ex. : point final qui entraîne la pause définitive ou temporaire). Certains organisateurs textuels séquentiels (d'une part, d'autre part; d'abord, ensuite, enfin; non seulement, mais encore) comportent aussi des affordances susceptibles de modifier le comportement en lecture. Par exemple, à la perception d'un d'abord, un lecteur pourra interrompre sa lecture normale de manière à rechercher le deuxième (ensuite) ou le troisième élément attendu (enfin) et poursuivre là sa lecture.

$\mathrm{Au}$ terme de la présentation des différents types d'affordances de notre modèle, il nous semble pertinent de réintroduire le concept de programmation de la lecture évoqué en introduction et pertinent à tous les autres niveaux de la typologie. Les propriétés des affordances identifiées dans notre typologie semblent indiquer que ces indices sont probablement en mesure de subordonner le traitement psycholinguistique à la perception de certaines affordances. Tout au long de son parcours de lecture, le lecteur serait guidé par les affordances décrites plus haut, et ce sont ces dernières qui seraient les principaux marqueurs qui définiraient l'entrée dans un processus de lecture au sens psycholinguistique. Les affordances, de notre point de vue, aideraient le lecteur à programmer sa lecture (de façon consciente et inconsciente) et le guideraient en ce sens tout au long de son parcours perceptuel du document et du texte. Il y aurait donc des affordances qui favoriseraient l'entrée en lecture au sens psycholinguistique et d'autres qui induiraient la suspension de la lecture.

\section{Conclusion}

Notre typologie n'est pas encore développée à son extension maximale et elle n'est présentée ici qu'au terme d'une phase exploratoire, certes féconde, mais limitée. Si le développement des affordances plus macroscopiques (5.1, 5.2 et 5.3) est peut-être moins susceptible de contribuer directement à la compréhension des interactions avec le texte lui-même (pris dans son sens étroit), objet plus naturel pour la rédactologie, il permettrait néanmoins un enrichissement écologique (au sens gibsonien) du modèle de lecture. Cette ouverture pourrait contribuer à repositionner le point de vue classique sur le texte (et la mise en texte) et ainsi autoriser à considérer le texte professionnel non pas seulement comme un artéfact langagier mais aussi comme un objet du monde à concevoir par-delà le paradigme linguistique et langagier. Cette perspective ouvrirait à la 
rédactologie les portes des sciences de la conception (design), discipline susceptible de nourrir de façon très positive la science de la conception/rédaction des textes professionnels utilitaires. Les affordances plus microscopiques de notre typologie (5.4, 5.5 et 5.6) devront être développées davantage, c'est une évidence, au point où même des ajouts de sous-types sont envisageables étant donné la grande variété des phénomènes observés.

Si la typologisation des affordances constitue un pas en avant par rapport à leur simple inventaire basé sur des observations, elle demeure malgré tout insuffisante compte tenu des vides épistémologiques constatés en rédactologie (Labasse, 2002, 2006). Néanmoins, cette typologie des affordances pourrait servir de base à un modèle de réception du document/texte utilitaire, modèle qui devrait, d'une part, subordonner les modèles linguistiques et psycholinguistiques existants et ainsi permettre à la rédactologie de faire un saut épistémologique, voire un changement de paradigme. D'autre part, ce modèle à produire pourrait être subordonné à des modèles plus généraux, comme celui de la théorie de l'activité (Kaptelinin \& Nardi, 2012b). L'intérêt de recourir à la théorie de l'activité pour positionner une commande d'écriture, par exemple, ou pour analyser un texte déjà produit se trouve à notre avis dans la possibilité d'intégrer une lecture donnée dans une chaîne d'activités dirigées vers un but plus large que celui de "simplement" réussir la lecture dudit texte. Cette prise en compte de l'activité humaine au sens large permettrait même un enrichissement du concept d'efficacité communicationnelle (Clerc \& Beaudet, 2008), peut-être même la possibilité de mieux structurer ce concept et, en bout de ligne, une façon plus complète d'en mesurer la réussite. En effet, l'usage d'un tel modèle permettrait de venir positionner la lecture non plus dans son seul spectre psycholinguistique mais aussi dans une perspective d'actions et d'activités humaines orientées vers des objectifs plus fondamentaux.

\section{BIBLIOGRAPHIE}

APCI, Designers interactifs, Mov'eo \& Systematic (2013). Le design des interfaces numériques en 170 mots-clés. Des interactions homme-machine au design interactif. Paris : Dunod.

CARon, J. (2008) [1989]. Précis de psycholinguistique, $2^{\mathrm{e}}$ éd. Paris : Presses universitaires de France. CHEMERo, A. (2003). « An Outline of a Theory of Affordances ». Ecological Psychology 15(2), p. 181-195.

CLERC, I. \& BEAUDET, C. (dirs) (2008). Langue, médiation et efficacité communicationnelle. Québec : Presses de l'Université Laval.

CLERC, I. \& KAVANAGH, É. (2006). De la lettre à la page Web : savoir communiquer avec le grand public. Québec : Publications du Québec.

DILLON, A. (2004). Designing Usable Electronic Text, $2^{\mathrm{e}}$ éd. Boca Raton: CRC Press.

ERLHOFF, M. \& MARShALl, T. (dirs) (2008). Design Dictionary. Perspectives on Design Terminology. Bâle: Birkhäuser. 
FORTIN, C. \& ROUSSEAU, R. (2012). Psychologie cognitive. Une approche de traitement de l'information. Québec : Presses de l'Université du Québec.

GERO, J. S. \& KANNENGIESSER, U. (2012). « Representational Affordances in Design, with Examples from Analogy Making and Optimization ». Research in Engineering Design 23(3), p. 235-249.

GIBSON, E. J. (2000). « Where is the Information for Affordances?». Ecological Psychology 12(1), p. 53-56.

GIBSON, J. J. (1979). The Ecological Approach to Visual Perception. New York: Taylor \& Francis.

JONES, K. S. (2003). « What is an Affordance? ». Ecological Psychology 15(2), p. 107-114.

KAPTELININ, V. \& NARDI, B. (2012a). «Affordances in HCI: Toward a Mediated Action Perspective ». Proceedings of the SIGCHI Conference on Human Factors in Computing Systems (CHI 2012), p. 967-976.

KAPTELININ, V. \& NARDI, B. (2012b). Activity Theory in HCI. Fundamentals and Reflections, Morgan \& Claypool.

LABASSE, B. (2002). «Entre déficit épistémologique et défi procédural : une discipline en souffrance d'expertise ». Technostyle 18(1), p. 95-116.

LABASSE, B. (2006) [2002]. La communication écrite. Une matière en quête de substance, $2^{\mathrm{e}}$ éd. Lyon : CECP Éditions.

LIDWELL, W., HOLDEN, K. \& BUTLER, J. (2003). Universal Principles of Design. 100 Ways to Enhance Usability, Influence Perception, Increase Appeal, Make Better Design Decisions, and Teach Through Design. Beverly : Rockport.

LIDWELl, W., HOLDEN, K. \& BUTLER, J. (2015). The Pocket Universal Principles of Design. 150 Essentials Tools for Architects, Artists, Designers, Developpers, Engineers, Inventors, and Makers. Beverly: Rockport.

MACE, W. M. (2015) [1979]. « Introduction to the Classic Edition ». In: Gibson, J. J., The Ecological Approach to Visual Perception. New York: Taylor \& Francis, p. xvii-xxix.

MAIER, J. (2011). Affordance Based Design. Theoretical Foundations and Practical Applications. Saarbrücken: VDM Verlag Dr. Müller.

MARIN, B. \& LEGROS, D. (2008). Psycholinguistique cognitive. Lecture, compréhension et production de texte. Bruxelles : De Boeck.

MICHAELS, C. F. (2003). « Affordances: Four Points of Debate ». Ecological Psychology 15(2), p. 135-148.

NIELSEN, J. (2007). « Banner Blindness: Old and New Findings ». En ligne : http:// www.nngroup.com/articles/banner-blindness-old-and-new-findings/.

NORMAN, D. A. (1999). «Affordance, Conventions, and Design ». Interactions 6(3), p. 38-43. - (2002) [1988]. The Design of Everyday Things. New York: Basic Books.

OQLF-GDT (1995). « Affordance, fiche terminologique ». In: Le grand dictionnaire terminologique (GDT). Office québécois de la langue française. En ligne : http://www.granddictionnaire.com/ ficheOqlf.aspx?Id_Fiche=17015787.

SOEGAARD, M. (2003). « Affordances ». In: Interaction Design Foundation. En ligne : http:// www.interaction-design.org/encyclopedia/affordances.html.

STILL, J. D. \& DARK, V. J. (2013). « Cognitively Describing and Designing Affordances ». Design Studies 34(3), p. 285-301. 
STOFFREgen, T. A. (2003). « Affordances are Enough: Reply to Chemero et al. (2003) ». Ecological

Psychology 15(1), p. 29-36.

STOFFREGEN, T. A. (2004). « Breadth and Limits of the Affordance Concept ». Ecological Psychology 16 (1), p. 79-85.

WEINSCHENK, S. M. (2011). 100 Things Every esigner Needs to Know About People. Berkeley: New Riders.

\section{NOTES}

1. Gibson avait anticipé le concept d'affordance dans certaines de ses communications préliminaires de la fin des années 1960 (les purple perils, voir Mace, 2015 et www.trincoll.edu/depts/ecopsyc/perils/).

\section{RÉSUMÉS}

Le concept d'affordance a été introduit par James J. Gibson dans le cadre de sa psychologie écologique en 1979. Les affordances sont des propriétés de l'environnement et des objets qui s'y trouvent. Lorsqu'elles sont perçues, ces propriétés permettent à l'animal - à l'humain - de savoir comment il peut agir dans un contexte donné. Omniprésentes, dans le monde et dans les objets du monde, ces propriétés concernent donc aussi tous les artéfacts destinés à la lecture, qu'il s'agisse d'un roman, d'une page Web, d'un formulaire fiscal, etc. En ce sens, les affordances doivent intéresser la rédactologie parce qu'elles influencent à divers degrés les interactions avec les documents et les textes en circulation dans notre société. Dans une logique exploratoire, nous proposons six types d'affordances qui nous semblent caractériser tous les documents et les textes (imprimés et numériques) susceptibles d'exister : les affordances gibsoniennes, les affordances de lisibilité fondamentale, les affordances linguistiques fondamentales, les affordances du genre, les affordances des zones informationnelles et les affordances microstructurelles. Les types proposés s'inscrivent dans un mouvement perceptuel allant des affordances macroscopiques vers les affordances microscopiques, mais toujours en marge du processus de lecture psycholinguistique.

\section{INDEX}

Mots-clés : affordance, typologie, lecture, rédactologie, design de communication

\section{AUTEURS}

\section{ÉRIC KAVANAGH}

Université Laval, École de design, Canada 


\section{JACYNTHE ROBERGE}

Université Laval, École de design, Canada

\section{ISABELLE SPERANO}

Université Laval, École de design, Canada 